\title{
SHARP $L^{2} \rightarrow L^{q}$ BOUNDS ON SPECTRAL PROJECTORS FOR LOW REGULARITY METRICS
}

\author{
HART F. SMITH
}

\begin{abstract}
We establish $L^{2} \rightarrow L^{q}$ mapping bounds for unit-width spectral projectors associated to elliptic operators with $C^{s}$ coefficients, in the case $1 \leq s \leq 2$. Examples of Smith-Sogge [6] show that these bounds are best possible for $q$ less than the critical index. We also show that $L^{\infty}$ bounds hold with the same exponent as in the case of smooth coefficients.
\end{abstract}

\section{Introduction}

The goal of this paper is to study the $L^{p}$ norms of eigenfunctions, and approximate eigenfunctions, of elliptic second order differential operators with low regularity coefficients, on compact manifolds without boundary. We consider the eigenvalues $-\lambda^{2}$ and eigenfunctions $\phi$ for an equation

$$
d^{*}(a d \phi)+\lambda^{2} \rho \phi=0 .
$$

Here we assume $\rho>0$ is a real, positive measurable function, and $a_{x}: T_{x}^{*}(M) \rightarrow$ $T_{x}(M)$ is the transformation associated to a real symmetric form on $T_{x}^{*}(M)$, also strictly positive and measurable in $x$. The manifold $M$ and volume form $d x$ are assumed smooth, and $d^{*}$ is the transpose of the differential $d$ with respect to $d x$. This setting includes the most general elliptic second order operator on $M$, assumed selfadjoint with respect to some measurable volume form $\rho d x$, and assumed to annihilate constants, and hence of the form $\rho^{-1} d^{*} a d$. For limited regularity $a$ and $\rho$ we pose the problem as above to avoid domain considerations.

If we consider the real quadratic forms

$$
Q_{0}(f, g)=\int_{M} f g \rho d x, \quad Q_{1}(f, g)=Q_{0}(f, g)+\int_{M} a(d f, d g) d x,
$$

then

$$
Q_{0}(f, f)=\|f\|_{L^{2}(M, \rho d x)}^{2}, \quad Q_{1}(f, f) \approx\|f\|_{H^{1}(M)}^{2},
$$

hence $Q_{0}$ is compact relative to $Q_{1}$ by Rellich's lemma. By the standard argument of simultaneously diagonalizing $Q_{0}$ and $Q_{1}$, there exists a complete orthonormal basis $\phi_{j}$ for $L^{2}(M, \rho d x)$ consisting of eigenfunctions for (1), with $\lambda_{j} \rightarrow \infty$.

The object of this paper is to establish bounds on the $L^{2} \rightarrow L^{q}$ operator norm of the unit-width spectral projectors for (1). Let $\Pi_{\lambda}$ be the projection of $L^{2}(M, \rho d x)$ onto the subspace spanned by the eigenfunctions of (1) for which $\lambda_{j} \in[\lambda, \lambda+1]$. In

Received by the editors April 3, 2006.

The author was supported in part by NSF grants DMS-0140499 and DMS-0354668. 
the case that the coefficients $\rho$ and $a$ are $C^{\infty}$, the following estimates hold, and are best possible in terms of the exponent of $\lambda$,

$$
\begin{array}{cl}
\left\|\Pi_{\lambda} f\right\|_{L^{q}(M)} \leq C \lambda^{\frac{n-1}{2}\left(\frac{1}{2}-\frac{1}{q}\right)}\|f\|_{L^{2}(M)}, & 2 \leq q \leq q_{n}, \\
\left\|\Pi_{\lambda} f\right\|_{L^{q}(M)} \leq C \lambda^{n\left(\frac{1}{2}-\frac{1}{q}\right)-\frac{1}{2}}\|f\|_{L^{2}(M)}, & q_{n} \leq q \leq \infty,
\end{array}
$$

where

$$
q_{n}=\frac{2(n+1)}{n-1}
$$

For $C^{\infty}$ metrics the estimates at $q=q_{n}$ are due to Sogge [8]. The estimate for $q=\infty$ is related to the spectral counting remainder estimates of Avakumović-LevitanHörmander; it can also be obtained from Sogge's estimate by Sobolev embedding. The case $q=2$ is of course trivial, and all other values of $q$ follow from these endpoints by interpolation.

In [5], both estimates (2) and (3) were established on the full range of $q$ for the case that both $a$ and $\rho$ are of class $C^{1,1}$.

On the other hand, Smith-Sogge [6] and Smith-Tataru [7] constructed examples, for each $0<s<2$, of functions $a$ and $\rho$ with coefficients of class $C^{s}$ (Lipschitz in case $s=1$ ) for which there exist eigenfunctions $\phi_{\lambda}$ such that for all $q \geq 2$

$$
\left\|\phi_{\lambda}\right\|_{L^{q}(M)} \geq C \lambda^{\frac{n-1}{2}\left(\frac{1}{2}-\frac{1}{q}\right)(1+\sigma)}\left\|\phi_{\lambda}\right\|_{L^{2}(M)},
$$

where $C>0$ is independent of $\lambda$, and where

$$
\sigma=\frac{2-s}{2+s}
$$

For $2<q<\frac{2\left(n+2 s^{-1}\right)}{n-1}$, this shows that the spectral projection estimates for $C^{s}$ metrics with $s<2$ can be strictly worse than in the $C^{2}$ case.

In this paper, we consider the case of coefficients $a$ and $\rho$ of class $C^{s}$ for $1 \leq s<2$ (Lipschitz in case $s=1$.) We start by establishing the following bound, which by the examples of [6] is best possible on the indicated range of $q$.

Theorem 1. Assume that the coefficients a and $\rho$ are either of class $C^{s}$ for some $1<s<2$, or Lipschitz class if $s=1$. Let $\Pi_{\lambda}$ denote the $L^{2}$-projection onto the subspace spanned by eigenfunctions of (1) with $\lambda_{j} \in[\lambda, \lambda+1]$. Then

$$
\left\|\Pi_{\lambda} f\right\|_{L^{q}(M)} \leq C \lambda^{\frac{n-1}{2}\left(\frac{1}{2}-\frac{1}{q}\right)(1+\sigma)}\|f\|_{L^{2}(M)}, \quad 2 \leq q \leq q_{n} .
$$

Applying Sobolev embedding to the estimate at $q=q_{n}$ would not yield the correct bound for $q=\infty$. However, the proof of Theorem 1 also yields no-loss estimates on small sets. Precisely, we will establish the following local estimate, with constant uniform over the balls $B$.

Theorem 2. Let $B_{R} \subset M$ be a ball of radius $R=\lambda^{-\sigma}$. Then under the same conditions as Theorem 1

$$
\left\|\Pi_{\lambda} f\right\|_{L^{q}\left(B_{R}\right)} \leq C \lambda^{n\left(\frac{1}{2}-\frac{1}{q}\right)-\frac{1}{2}}\|f\|_{L^{2}(M)}, \quad q_{n} \leq q \leq \infty .
$$


Interpolating with the trivial $L^{2}$ estimate establishes the estimate (2) on such balls $B_{R}$. Since the constant $C$ in (4) is uniform for all balls $B_{R}$, we obtain the same global $L^{2} \rightarrow L^{\infty}$ mapping properties in the case of Lipschitz coefficients as in the case of smooth coefficients,

$$
\left\|\Pi_{\lambda} f\right\|_{L^{\infty}(M)} \leq C \lambda^{\frac{n-1}{2}}\|f\|_{L^{2}(M)} .
$$

A corollary of this result is the Hörmander multiplier theorem on compact manifolds for functions of elliptic operators with Lipschitz coefficients, as shown by results of Duong-Ouhabaz-Sikora [1], section 7.2. We note that, in related work, Ivrii [2] has obtained the sharp spectral counting remainder estimate for operators with coefficients of regularity slightly stronger than Lipschitz.

The proof of Theorem 2 that we will present requires that $q$ be not too large, but in all dimensions works for $q=q_{n}$. We therefore show here how heat kernel estimates permit us to deduce (4) for all $q \geq q_{n}$ from the case $q=q_{n}$. For this, let $H_{\lambda}$ denote the heat kernel at time $\lambda^{-2} \leq 1$ for the diffusion system associated to (1). By Theorem 6.3 of Saloff-Coste [4], the integral kernel $h_{\lambda}$ of $H_{\lambda}$ satisfies

$$
\left|h_{\lambda}(x, y)\right| \leq C \lambda^{n} \exp \left(-c \lambda^{2} d(x, y)^{2}\right) .
$$

By Young's inequality, then for $q_{n} \leq q \leq \infty$

$$
\begin{aligned}
\left\|\Pi_{\lambda} f\right\|_{L^{q}\left(B_{R}\right)} & \leq C \lambda^{n\left(\frac{1}{q_{n}}-\frac{1}{q}\right)}\left\|H_{\lambda}^{-1} \Pi_{\lambda} f\right\|_{L^{q_{n}}\left(B_{R}^{*}\right)}+C_{N} \lambda^{-N}\left\|H_{\lambda}^{-1} \Pi_{\lambda} f\right\|_{L^{2}\left(M \backslash B_{R}^{*}\right)} \\
& \leq C \lambda^{n\left(\frac{1}{2}-\frac{1}{q}\right)-\frac{1}{2}}\|f\|_{L^{2}(M)}
\end{aligned}
$$

where we use (4) at $q=q_{n}$ with $B_{R}$ replaced by its double $B_{R}^{*}$, and the fact that $\left\|H_{\lambda}^{-1} \Pi_{\lambda} f\right\|_{L^{2}} \approx\left\|\Pi_{\lambda} f\right\|_{L^{2}}$ since $\exp \left(\lambda_{j}^{2} / \lambda^{2}\right) \approx 1$ for $\lambda_{j} \in[\lambda, \lambda+1]$.

If we interpolate the estimate of Theorem 1 at $q=q_{n}$ with the estimate (5), then we obtain the following.

Corollary 3. Under the same conditions as Theorem 1

$$
\left\|\Pi_{\lambda} f\right\|_{L^{q}(M)} \leq C \lambda^{n\left(\frac{1}{2}-\frac{1}{q}\right)-\frac{1}{2}+\frac{\sigma}{q}}\|f\|_{L^{2}(M)}, \quad q_{n} \leq q \leq \infty .
$$

For $q_{n}<q<\infty$, however, the exponent is strictly larger than that predicted by the examples of [6]. It is not currently known what the sharp exponent is for this range.

The key idea in our proof is that a $C^{s}$ function is well approximated on sets of diameter $R=\lambda^{-\sigma}$ by a $C^{2}$ function, up to an error which is suitably bounded when dealing with eigenfunctions localized to frequency $\lambda$. In effect, rescaling by $R$ reduces matters to a $C^{2}$ situation, where no-loss estimates hold. The loss of $\lambda^{\frac{\sigma}{q}}$ comes from adding up the bounds over $\approx R^{-1}$ disjoint sets.

This scaling parameter $R$ occurs in the examples of Smith-Sogge [6] and SmithTataru [7]. The idea of scaling by $R$ to prove $L^{p}$ estimates was first used by Tataru in [9], to establish Strichartz-type estimates for time-dependent wave equations with $C^{s}$ coefficients, yielding improved existence theorems for a class of quasilinear hyperbolic equations.

Notation. By a $C^{s}$ function on $\mathbb{R}^{n}$, for $1<s \leq 2$ we understand a continuously differentiable function $f$ such that

$$
\|f\|_{C^{s}}=\|f\|_{L^{\infty}\left(\mathbb{R}^{n}\right)}+\|d f\|_{L^{\infty}\left(\mathbb{R}^{n}\right)}+\sup _{h \in \mathbb{R}^{n}}|h|^{1-s}\|d f(\cdot+h)-d f(\cdot)\|_{L^{\infty}\left(\mathbb{R}^{n}\right)}<\infty .
$$


Thus, $C^{s}$ coincides with $C^{1, s-1}$ for $s \in(1,2]$. For $s=1$, we use $C^{1}$ to mean Lipschitz. For $0<s<1$ we take $C^{s}$ to be the standard Holder class.

We use $d$ to denote the differential taking functions to covector fields, and $d^{*}$ its adjoint with respect to $d x$. When working on $\mathbb{R}^{n}, d=\left(\partial_{1}, \ldots, \partial_{n}\right)$, and $d^{*}$ is the standard divergence operator.

The notation $A \lesssim B$ means $A \leq C B$, where $C$ is a constant that depends only on the $C^{s}$ norm of $a$ and $\rho$, as well as on universally fixed quantities, such as the manifold $M$ and the non-degeneracy of $a$ and $\rho$. In particular, $C$ can be taken to depend continuously on $a$ and $\rho$ in the $C^{s}$ norm, so our estimates are uniform under small $C^{s}$ perturbations of $a$ and $\rho$.

\section{Scaling Arguments}

Our starting point is the following square-function estimate for solutions to the Cauchy problem. For $C^{\infty}$ coefficients this was established by Mockenhaupt-SeegerSogge [3]. The version we need for $C^{1,1}$ metrics is Theorem 1.3 of [5]. That theorem was stated under the condition $F=0$ and for coefficients which are constant for large $x$, but these conditions are easily dropped by the Duhamel principle and a partition of unity argument.

Theorem 4. Suppose that $a$ and $\rho$ are defined globally on $\mathbb{R}^{n}$, and that

$$
\left\|a^{i j}-\delta^{i j}\right\|_{C^{1,1}\left(\mathbb{R}^{n}\right)}+\|\rho-1\|_{C^{1,1}\left(\mathbb{R}^{n}\right)} \leq c_{0},
$$

where $c_{0}$ is a small constant depending only on $n$. Let $u$ solve the Cauchy problem

$$
\rho(x) \partial_{t}^{2} u(t, x)-d^{*}(a(x) d u(t, x))=F(t, x), \quad u(0, x)=u_{0}(x), \quad \partial_{t} u(0, x)=u_{1}(x) .
$$

Then

$$
\|u\|_{L_{x}^{q_{n}} L_{t}^{2}\left(\mathbb{R}^{n} \times[-1,1]\right)} \lesssim\left\|u_{0}\right\|_{H^{\frac{1}{q_{n}}}}+\left\|u_{1}\right\|_{H^{\frac{1}{q_{n}}-1}}+\|F\|_{L_{t}^{1} H^{\frac{1}{q_{n}}-1}}
$$

We first deduce the following corollary which is more useful for our purposes.

Corollary 5. Suppose that $f$ satisfies an equation on $\mathbb{R}^{n}$ of the form

$$
d^{*}(a d f)+\mu^{2} \rho f=d^{*} g_{1}+g_{2} .
$$

If $a$ and $\rho$ satisfy the condition of Theorem 4, then

$$
\|f\|_{L^{q_{n}}} \lesssim \mu^{\frac{1}{q_{n}}}\left(\|f\|_{L^{2}}+\mu^{-1}\|d f\|_{L^{2}}+\left\|g_{1}\right\|_{L^{2}}+\mu^{-1}\left\|g_{2}\right\|_{L^{2}}\right) .
$$

Proof. Let $S_{r}=S_{r}(D)$ denote a smooth cutoff on the Fourier transform side to frequencies of size $|\xi| \leq r$. Let $a_{\mu}=S_{c^{2} \mu} a$, for $c$ to be chosen suitably small. Then

$$
\left\|\left(a-a_{\mu}\right) d f\right\|_{L^{2}} \lesssim c^{-2} \mu^{-1}\|d f\|_{L^{2}}, \quad \mu^{2}\left\|\left(\rho-\rho_{\mu}\right) f\right\|_{L^{2}} \lesssim c^{-2} \mu\|f\|_{L^{2}},
$$

and thus we may replace $a$ and $\rho$ by $a_{\mu}$ and $\rho_{\mu}$ at the expense of absorbing the above two terms into $g_{1}$ and $g_{2}$, which does not change the size of the right hand side of (7).

Next, let $f_{<\mu}=S_{c \mu} f$. Since

$$
\left\|\left[S_{c \mu}, a_{\mu}\right]\right\|_{L^{2} \rightarrow L^{2}} \lesssim(c \mu)^{-1}
$$

and similarly for $\left[S_{c \mu}, \rho_{\mu}\right]$, we can absorb the commutator terms into $g_{1}$ and $g_{2}$, and since all terms are localized to frequencies less than $\mu$ we can write

$$
d^{*}\left(a_{\mu} d f_{<\mu}\right)+\mu^{2} \rho_{\mu} f_{<\mu}=g_{<\mu},
$$


where

$$
\left\|g_{<\mu}\right\|_{L^{2}} \lesssim \mu\|f\|_{L^{2}}+\|d f\|_{L^{2}}+\mu\left\|g_{1}\right\|_{L^{2}}+\left\|g_{2}\right\|_{L^{2}}
$$

Since $\left\|d^{*}\left(a_{\mu} d f_{<\mu}\right)\right\|_{L^{2}} \lesssim(c \mu)^{2}\left\|f_{<\mu}\right\|_{L^{2}}$, for $c$ suitably small the $L^{2}$ norm of the left hand side of (8) is comparable to $\mu^{2}\left\|f_{<\mu}\right\|_{L^{2}}$, hence we have

$$
\left\|f_{<\mu}\right\|_{L^{2}} \lesssim \mu^{-1}\left(\|f\|_{L^{2}}+\mu^{-1}\|d f\|_{L^{2}}+\left\|g_{1}\right\|_{L^{2}}+\mu^{-1}\left\|g_{2}\right\|_{L^{2}}\right)
$$

Sobolev embedding now implies (7) if $f$ is replaced on the left hand side by $f_{<\mu}$. In fact there is a gain of $\mu^{-\frac{1}{2}}$, since $\frac{1}{q_{n}}=n\left(\frac{1}{2}-\frac{1}{q_{n}}\right)-\frac{1}{2}$.

If we let $f_{>\mu}=f-S_{c^{-1} \mu} f$, then similar arguments let us write

$$
d^{*}\left(a_{\mu} d f_{>\mu}\right)+\mu^{2} \rho_{\mu} f_{>\mu}=d^{*} g_{>\mu}
$$

where now $g_{>\mu}$, like $f_{>\mu}$, is frequency localized to frequencies larger than $c^{-1} \mu$, and

$$
\left\|g_{>\mu}\right\|_{L^{2}} \lesssim\|f\|_{L^{2}}+\mu^{-1}\|d f\|_{L^{2}}+\left\|g_{1}\right\|_{L^{2}}+\mu^{-1}\left\|g_{2}\right\|_{L^{2}}
$$

Taking the inner product of both sides of (9) against $f_{>\mu}$ yields

$$
\left\|d f_{>\mu}\right\|_{L^{2}}^{2}-4 \mu^{2}\left\|f_{>\mu}\right\|_{L^{2}}^{2} \lesssim\left\|g_{>\mu}\right\|_{L^{2}}\left\|d f_{>\mu}\right\|_{L^{2}}
$$

and by the frequency localization of $f_{>\mu}$ we obtain

$$
\left\|f_{>\mu}\right\|_{H^{1}} \lesssim\|f\|_{L^{2}}+\mu^{-1}\|d f\|_{L^{2}}+\left\|g_{1}\right\|_{L^{2}}+\mu^{-1}\left\|g_{2}\right\|_{L^{2}}
$$

Since $n\left(\frac{1}{2}-\frac{1}{q_{n}}\right)=\frac{1}{q_{n}}+\frac{1}{2} \leq 1$, Sobolev embedding yields (7) if $f$ is replaced on the left hand side by $f_{>\mu}$. As above, there is in fact a gain of $\mu^{-\frac{1}{2}}$ for this term.

We now let $f_{\mu}=S_{c^{-1} \mu} f-S_{c \mu} f$, and as above write

$$
d^{*}\left(a_{\mu} d f_{\mu}\right)+\mu^{2} \rho_{\mu} f_{\mu}=g_{\mu}
$$

where now $f_{\mu}$ and $g_{\mu}$ are localized to frequencies comparable to $\mu$, and

$$
\left\|g_{\mu}\right\|_{L^{2}} \lesssim \mu\|f\|_{L^{2}}+\|d f\|_{L^{2}}+\mu\left\|g_{1}\right\|_{L^{2}}+\left\|g_{2}\right\|_{L^{2}}
$$

Setting $u(t, x)=\cos (\mu t) f_{\mu}(x)$, we apply (6) to deduce

$$
\left\|f_{\mu}\right\|_{L^{q}} \lesssim \mu^{\frac{1}{q_{n}}}\left(\left\|f_{\mu}\right\|_{L^{2}}+\mu^{-1}\left\|g_{\mu}\right\|_{L^{2}}\right)
$$

which yields (7) for this term.

Remark. For future use, we note that in the proof of Corollary 5 the assumption that $a \in C^{1,1}$ was used only at the last step, in order to deduce that (6) holds. The commutator and approximation bounds require only that $a$ and $\rho$ be Lipschitz. In particular, the bounds on $f_{<\mu}$ and $f_{>\mu}$ hold for Lipschitz $a$ and $\rho$.

Corollary 6. Let $Q$ be a unit cube and $Q^{*}$ its double. Suppose that a and $\rho$ are bounded and measurable, and that there exist $C^{1,1}$ functions $\tilde{a}$ and $\tilde{\rho}$ satisfying the conditions of Theorem 4 such that

$$
\|a-\tilde{a}\|_{L^{\infty}\left(Q^{*}\right)}+\|\rho-\tilde{\rho}\|_{L^{\infty}\left(Q^{*}\right)} \leq \mu^{-1}
$$

Suppose that on $Q^{*}$ we have

$$
d^{*}(a d f)+\mu^{2} \rho f=d^{*} g_{1}+g_{2}
$$

Then

$$
\|f\|_{L^{q_{n}}(Q)} \lesssim \mu^{\frac{1}{q_{n}}}\left(\|f\|_{L^{2}\left(Q^{*}\right)}+\mu^{-1}\|d f\|_{L^{2}\left(Q^{*}\right)}+\left\|g_{1}\right\|_{L^{2}\left(Q^{*}\right)}+\mu^{-1}\left\|g_{2}\right\|_{L^{2}\left(Q^{*}\right)}\right)
$$


The constant in the inequality is uniform for $\mu \geq 1$.

Proof. Let $\phi$ be a smooth function, equal to 1 on $Q$ and supported in $Q^{*}$. Then

$$
\begin{aligned}
d^{*}(a d(\phi f))+\mu^{2} \rho(\phi f) & =d^{*}\left[(a d \phi) f+\phi g_{1}\right]+\left[(a d \phi) \cdot d f-(d \phi) \cdot g_{1}+\phi g_{2}\right] \\
& =d^{*} \tilde{g}_{1}+\tilde{g}_{2}
\end{aligned}
$$

where for $\mu \geq 1$

$$
\left\|\tilde{g}_{1}\right\|_{L^{2}}+\mu^{-1}\left\|\tilde{g}_{2}\right\|_{L^{2}} \lesssim\|f\|_{L^{2}\left(Q^{*}\right)}+\mu^{-1}\|d f\|_{L^{2}\left(Q^{*}\right)}+\left\|g_{1}\right\|_{L^{2}\left(Q^{*}\right)}+\mu^{-1}\left\|g_{2}\right\|_{L^{2}\left(Q^{*}\right)}
$$

One may similarly absorb $(a-\tilde{a}) d(\phi f)$ into $\tilde{g}_{1}$, and $\mu^{2}(\rho-\tilde{\rho})(\phi f)$ into $\tilde{g}_{2}$. The result now follows from (7).

Corollary 7. Suppose that $a$ and $\rho$ are of class $C^{s}$, with $0 \leq s \leq 2$, and that

$$
\left\|a^{i j}-\delta^{i j}\right\|_{C^{s}\left(\mathbb{R}^{n}\right)}+\|\rho-1\|_{C^{s}\left(\mathbb{R}^{n}\right)} \leq c_{0},
$$

where $c_{0}$ is a small constant depending only on $n$.

Suppose that $R=\lambda^{-\sigma}$, where $\sigma=\frac{2-s}{2+s}$ and $\lambda \geq 1$. Assume $Q_{R}$ is a cube of sidelength $R, Q_{R}^{*}$ is its double, and on $Q_{R}^{*}$ the following equation holds

$$
d^{*}(a d f)+\lambda^{2} \rho f=d^{*} g_{1}+g_{2}
$$

Then

$$
\begin{aligned}
\|f\|_{L^{q_{n}}\left(Q_{R}\right)} \lesssim R^{-\frac{1}{2}} \lambda^{\frac{1}{q_{n}}}\left(\|f\|_{L^{2}\left(Q_{R}^{*}\right)}+\lambda^{-1}\|d f\|_{L^{2}\left(Q_{R}^{*}\right)}\right. & \\
& \left.+R\left\|g_{1}\right\|_{L^{2}\left(Q_{R}^{*}\right)}+R \lambda^{-1}\left\|g_{2}\right\|_{L^{2}\left(Q_{R}^{*}\right)}\right) .
\end{aligned}
$$

Proof. We use the notation $f_{R}(x)=f(R x)$. Then, for $\mu=R \lambda=\lambda^{1-\sigma}$,

$$
d^{*}\left(a_{R} d f_{R}\right)+\mu^{2} \rho_{R} f_{R}=R d^{*} g_{1, R}+R^{2} g_{2, R}
$$

holds on $Q^{*}$, with $Q$ a unit cube. If $\tilde{a}=S_{\mu^{1 / 2}} a_{R}$, then

$$
\left\|\tilde{a}-a_{R}\right\|_{L^{\infty}} \lesssim \mu^{-\frac{1}{2} s} R^{s}\|a-I\|_{C^{s}}=c_{0} \mu^{-1}
$$

By the frequency localization, $\tilde{a}$ satisfies the conditions of Theorem 4 . We may thus apply Corollary 6 to yield

$$
\begin{aligned}
\left\|f_{R}\right\|_{L^{q_{n}}(Q)} \lesssim(R \lambda)^{\frac{1}{q_{n}}}\left(\left\|f_{R}\right\|_{L^{2}\left(Q^{*}\right)}+\lambda^{-1}\left\|(d f)_{R}\right\|_{L^{2}\left(Q^{*}\right)}\right. & \\
& \left.+R\left\|g_{1, R}\right\|_{L^{2}\left(Q^{*}\right)}+R \lambda^{-1}\left\|g_{2, R}\right\|_{L^{2}\left(Q^{*}\right)}\right)
\end{aligned}
$$

Recalling that $\frac{1}{q_{n}}=n\left(\frac{1}{2}-\frac{1}{q_{n}}\right)-\frac{1}{2}$, this yields the corollary after rescaling.

\section{Proof of Theorem 1}

The proof of Corollary 7 works for all $s \in[0,2]$, but the energy estimates of this section require that $a$ and $\rho$ be Lipschitz, hence we assume $s \geq 1$ for the remainder.

The projection $\Pi_{\lambda} f$ satisfies

$$
\begin{aligned}
\left\|d^{*}\left(\operatorname{ad}\left(\Pi_{\lambda} f\right)\right)+\lambda^{2} \rho \Pi_{\lambda} f\right\|_{L^{2}(M, \rho d x)} & \leq(2 \lambda+1)\left\|\Pi_{\lambda} f\right\|_{L^{2}(M, \rho d x)} \\
\left\|d \Pi_{\lambda} f\right\|_{L^{2}(M, \rho d x)} & \lesssim(\lambda+1)\left\|\Pi_{\lambda} f\right\|_{L^{2}(M, \rho d x)}
\end{aligned}
$$

hence Theorem 1 follows from showing that, if the following holds on $M$

$$
d^{*}(a d f)+\lambda^{2} \rho f=g
$$


then uniformly for $\lambda \geq 1$

$$
\|f\|_{L^{q_{n}}(M)} \lesssim \lambda^{\frac{1+\sigma}{q_{n}}}\left(\|f\|_{L^{2}(M)}+\lambda^{-1}\|d f\|_{L^{2}(M)}+\lambda^{-1}\|g\|_{L^{2}(M)}\right)
$$

Assume that (10) holds, and let $\phi$ be a $C^{2}$ bump function on $M$. Then

$$
d^{*}(a d(\phi f))+\lambda^{2} \rho \phi f=f d^{*}(a d \phi)+\langle a d \phi, d f\rangle+\phi g
$$

Absorbing the terms on the right into $g$ leaves the right hand side of (11) unchanged, hence by a partition of unity argument we may assume that $f$ is supported in a suitably small coordinate neighborhood on $M$.

We choose coordinate patches so that, in local coordinates, the conditions of Corollary 7 are satisfied after extending $a$ and $\rho$ to all of $\mathbb{R}^{n}$. Thus, we have an equation of the form (10) on $\mathbb{R}^{n}$, with $f$ and $g$ supported in a unit cube.

We next decompose $f=f_{<\lambda}+f_{>\lambda}+f_{\lambda}$ as in the proof of Corollary 5. As remarked following that proof, the bounds on $f_{<\lambda}$ and $f_{>\lambda}$ hold for $a$ and $\rho$ Lipschitz, hence we are reduced to considering $f_{\lambda}$, for which we have an equation

$$
d^{*}\left(a_{\lambda} d f_{\lambda}\right)+\lambda^{2} \rho_{\lambda} f_{\lambda}=g_{\lambda}
$$

where $a_{\lambda}$ and $\rho_{\lambda}$ are localized to frequencies smaller than $c^{2} \lambda$, and both $f_{\lambda}$ and $g_{\lambda}$ are localized to frequencies of size comparable to $\lambda$.

We then decompose $f_{\lambda}=\sum_{j=1}^{N} \Gamma_{j} f_{\lambda}$, where each $\Gamma_{j}=\Gamma_{j}(D)$ is an order 0 multiplier, with symbol $\Gamma_{j}(\xi)$ supported where $|\xi| \approx \lambda$ and in a cone of suitably small angle. It then suffices to bound each $\left\|\Gamma_{j} f_{\lambda}\right\|_{L^{q_{n}}(Q)}$ by the right hand side of (11). Without loss of generality we consider a term with $\Gamma(\xi)$ localized to a small cone about the $\xi_{1}$ axis.

We write

$$
d^{*}\left(a_{\lambda} d \Gamma f_{\lambda}\right)+\lambda^{2} \rho_{\lambda} \Gamma f_{\lambda}=\Gamma g_{\lambda}+d^{*}\left[a_{\lambda}, \Gamma\right] d f_{\lambda}+\lambda^{2}\left[\rho_{\lambda}, \Gamma\right] f_{\lambda}
$$

Simple commutator estimates show that the right hand side has $L^{2}$ norm bounded by $\lambda\|f\|_{L^{2}}+\|g\|_{L^{2}}$, hence we are reduced to establishing

$$
\|f\|_{L^{q_{n}}(Q)} \lesssim \lambda^{\frac{1+\sigma}{q_{n}}}\left(\|f\|_{L^{2}\left(\mathbb{R}^{n}\right)}+\lambda^{-1}\|d f\|_{L^{2}\left(\mathbb{R}^{n}\right)}+\lambda^{-1}\|g\|_{L^{2}\left(\mathbb{R}^{n}\right)}\right)
$$

for $f$ satisfying the equation

$$
d^{*}\left(a_{\lambda} d f\right)+\lambda^{2} \rho_{\lambda} f=g
$$

where $\widehat{f}(\xi)$ and $\widehat{g}(\xi)$ are localized to $|\xi| \approx \lambda$ and $\xi$ in a small cone about the $\xi_{1}$ axis.

By Corollary 7, for any cube $Q_{R}$ of sidelength $R=\lambda^{-\sigma}$, we have

$$
\|f\|_{L^{q_{n}}\left(Q_{R}\right)} \lesssim \lambda^{\frac{1}{q_{n}}}\left(R^{-\frac{1}{2}}\|f\|_{L^{2}\left(Q_{R}^{*}\right)}+R^{-\frac{1}{2}} \lambda^{-1}\|d f\|_{L^{2}\left(Q_{R}^{*}\right)}+R^{\frac{1}{2}} \lambda^{-1}\|g\|_{L^{2}\left(Q_{R}^{*}\right)}\right) \text {. }
$$

Let $S_{R}$ denote a slab of the form $\left\{x \in \mathbb{R}^{n}:\left|x_{1}-c\right| \leq R\right\}$. By summing over cubes $Q_{R}$ contained in $S_{R}$, and noting $R \leq 1$, we obtain

$$
\|f\|_{L^{q_{n}}\left(S_{R}\right)} \lesssim \lambda^{\frac{1}{q_{n}}}\left(R^{-\frac{1}{2}}\|f\|_{L^{2}\left(S_{R}^{*}\right)}+R^{-\frac{1}{2}} \lambda^{-1}\|d f\|_{L^{2}\left(S_{R}^{*}\right)}+\lambda^{-1}\|g\|_{L^{2}\left(S_{R}^{*}\right)}\right)
$$

We will show that

(15) $R^{-\frac{1}{2}}\left(\|f\|_{L^{2}\left(S_{R}^{*}\right)}+\lambda^{-1}\|d f\|_{L^{2}\left(S_{R}^{*}\right)}\right) \lesssim\|f\|_{L^{2}\left(\mathbb{R}^{n}\right)}+\lambda^{-1}\|d f\|_{L^{2}\left(\mathbb{R}^{n}\right)}+\lambda^{-1}\|g\|_{L^{2}\left(\mathbb{R}^{n}\right)}$

Given this, inequality (12) follows from (14) by adding over the $R^{-1}=\lambda^{\sigma}$ disjoint slabs that intersect $Q$. Also, the bound (13) implies the conclusion of Theorem 2 for $q=q_{n}$ (hence for all $q$ by the heat kernel arguments following that theorem.) 
We establish (15) by energy inequality arguments. Let $V$ denote the vector field

$$
V=2\left(\partial_{1} f\right) a_{\lambda} d f+\left(\lambda^{2} \rho_{\lambda} f^{2}-\left\langle a_{\lambda} d f, d f\right\rangle\right) \overrightarrow{e_{1}}
$$

Then

$$
d^{*} V=2\left(\partial_{1} f\right) g+\lambda^{2}\left(\partial_{1} \rho_{\lambda}\right) f^{2}-\left\langle\left(\partial_{1} a_{\lambda}\right) d f, d f\right\rangle
$$

Applying the divergence theorem on the set $x_{1} \leq r$ yields

$$
\int_{x_{1}=r} V_{1} d x^{\prime} \lesssim \lambda^{2}\|f\|_{L^{2}\left(\mathbb{R}^{n}\right)}^{2}+\|d f\|_{L^{2}\left(\mathbb{R}^{n}\right)}^{2}+\|g\|_{L^{2}\left(\mathbb{R}^{n}\right)}^{2}
$$

Since $a_{\lambda}$ and $\rho$ are pointwise close to the flat metric, we have pointwise that

$$
V_{1} \geq \frac{3}{4}\left|\partial_{1} f\right|^{2}+\frac{3}{4} \lambda^{2}|f|^{2}-\left|\partial_{x^{\prime}} f\right|^{2}
$$

The frequency localization of $\widehat{f}$ to $\left|\xi^{\prime}\right| \leq c \lambda$ yields

$$
\int_{x_{1}=r} V_{1} d x^{\prime} \geq \frac{1}{2} \int_{x_{1}=r}|d f|^{2}+\lambda^{2}|f|^{2} d x^{\prime}
$$

Integrating this over $r$ in an interval of size $R$ yields (15).

\section{References}

[1] X.T. Duong, E.M. Ouhabaz and A. Sikora, Plancherel-type estimates and sharp spectral multipliers, J. Funct. Anal. 196 (2002), 443-485.

[2] V. Ivrii, Sharp spectral asymptotics for operators with irregular coefficients, Internat. Math. Res. Notices 2000 no. 22, 1155-1166.

[3] G. Mockenhaupt, A. Seeger and C. Sogge, Local smoothing of Fourier integral operators and Carleson-Sjölin estimates, J. Amer. Math. Soc. 6 (1993), no. 1, 65-130.

[4] L. Saloff-Coste, Uniformly elliptic operators on Riemannian manifolds, J. Differential Geom. 36 (1992), 417-450.

[5] H. Smith, Spectral cluster estimates for $C^{1,1}$ metrics, Amer. Jour. Math., to appear.

[6] H. Smith and C. Sogge, On Strichartz and eigenfunction estimates for low regularity metrics, Math. Res. Lett. 1 (1994), 729-737.

[7] H. Smith and D. Tataru, Sharp counterexamples for Strichartz estimates for low regularity metrics, Math. Res. Lett. 9 (2002), 199-204.

[8] C. Sogge, Concerning the $L^{p}$ norm of spectral clusters for second-order elliptic operators on compact manifolds, J. Funct. Anal. 77 (1988), no. 1, 123-134.

[9] D. Tataru, Strichartz estimates for second order hyperbolic operators with nonsmooth coefficients. III., J. Amer. Math. Soc. 15 (2002), 419-442.

Department of Mathematics, University of Washington, Seattle, WA 98195

E-mail address: hart@math.washington.edu 